\title{
Humidity and Temperature Induced Changes in the Diffraction Efficiency and the Bragg Angle of Slanted Photopolymer-based Holographic Gratings
}

\author{
Tatsiana Mikulchyk \\ Technological University Dublin, Tatsiana.mikulchyk@tudublin.ie \\ James Walshe \\ Technological University Dublin, james.walshe@tudublin.ie \\ Dervil Cody \\ Technological University of Dublin, dervil.cody@tudublin.ie
}

See next page for additional authors

Follow this and additional works at: https://arrow.tudublin.ie/cieoart

Part of the Optics Commons

\section{Recommended Citation \\ Mikulchyk, T., Walshe, J., Cody, D., Martin, S. \& Naydenova, I. (2017). Humidity and temperature induced changes in the diffraction efficiency and the Bragg angle of slanted photopolymer-based holographic gratings. Sensor and Actuators, B.:Chemical, vol. 239, pg. 776-785. doi:10.1016/j.snb.2016.08.052}

This Article is brought to you for free and open access by the Centre for Industrial and Engineering Optics at ARROW@TU Dublin. It has been accepted for inclusion in Articles by an authorized administrator of ARROW@TU Dublin. For more information, please contact arrow.admin@tudublin.ie, aisling.coyne@tudublin.ie, gerard.connolly@tudublin.ie.

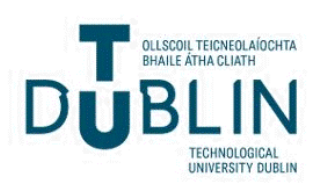




\section{Authors}

Tatsiana Mikulchyk, James Walshe, Dervil Cody, Suzanne Martin, and Izabela Naydenova 


\title{
Humidity and temperature induced changes in the diffraction efficiency and the Bragg angle of slanted photopolymer-based holographic gratings
}

\author{
Tatsiana Mikulchyk, James Walshe, Dervil Cody, Suzanne Martin, Izabela Naydenova* \\ Centre for Industrial and Engineering Optics, School of Physics, College of Sciences and Health, Dublin Institute of \\ Technology, Kevin Street, Dublin 8, Ireland \\ *e-mail: izabela.naydenova@dit.ie
}

This work explores the humidity and temperature response of volume phase slanted gratings recorded in photopolymers with varied chemical composition. Acrylamide and diacetone acrylamide were used as monomers and triethanolamine and $N$-phenylglycine were used as photoinitiators. The study demonstrates that the response of photopolymer-based holographic gratings to relative humidity $(\mathrm{RH})$ and temperature $(\mathrm{T})$ can be tuned by alteration of the photopolymer composition.

Humidity and temperature response of the holograms has been characterized by recording Bragg selectivity curves of transmission gratings and by monitoring the position of the maximum intensity in the spectral response of reflection gratings. Investigation of the humidity response in the range of $20-90 \% \mathrm{RH}$ reveals that photopolymers containing triethanolamine are more responsive to moisture than photopolymers containing $N$-phenylglycine and display significant sensitivity to relative humidity above $40 \%$. Full reversibility of humidity induced changes in gratings recorded in diacetone acrylamide-based photopolymer is confirmed at $\mathrm{RH} \leq 60 \%$. Exposure to $\mathrm{RH} \geq 70 \%$ leads to irreversible changes in these gratings.

The temperature response of slanted transmission gratings was investigated in the temperature range of $20-60{ }^{0} \mathrm{C}$. Exposure of the photopolymer layers containing triethanolamine to elevated temperature was found to cause layer shrinkage due to desorption of absorbed water. Sealed layers containing triethanolamine, however, demonstrated swelling due to the effect of thermal expansion. The photopolymer layers containing $N$-phenylglycine were found to be unresponsive to temperature changes below $30{ }^{\circ} \mathrm{C}$ and have sensitivity to temperature above $30{ }^{\circ} \mathrm{C}$.

Keywords: photopolymer; diacetone acrylamide; holographic grating; holographic sensor; sensitivity to relative humidity; sensitivity to temperature.

\section{INTRODUCTION}

Holographic gratings capable of responding to an analyte with an easily identifiable change in their optical properties have generated wide interest due to their possible application as holographic sensors. It has been demonstrated that holographic sensors can detect analytes in gas and in liquid phase as well as different physical stimuli such as pressure and magnetic fields ${ }^{1-12}$. Holographic sensors are considered a low-cost, lightweight, and disposable technology and have potential for application in different areas ranging from medical diagnostics to environmental sensing including the monitoring of environmental temperature and relative humidity ${ }^{10,12,13}$. In spite of the existing wide range of temperature and humidity sensors ${ }^{14,15}$, holographic sensors are of special interest as they can provide fast, real-time, reversible or irreversible, visual colorimetric or optical readouts.

The intrinsic properties of some photopolymers, such as their hygroscopic nature and thermal expansion, make them good candidates for the development of humidity and temperature holographic sensors. Recently, the humidity sensitivity of reflection gratings recorded in an acrylamide-based photopolymer has been demonstrated and characterized in the range of $5-80 \% \mathrm{RH}^{10,12,13}$. Reversible changes in fringe spacing due to water vapour absorption have been found to cause fully reversible variations in the diffracted light wavelength. The natural ability of the acrylamide-based photopolymer layer to swell or shrink at different levels of relative humidity was exploited for the design of a holographic humidity indicator ${ }^{10}$.

The response to humidity of unslanted transmission gratings in similar material has been investigated in the range of 20 $90 \% \mathrm{RH}$ with particular emphasis on irreversible changes caused by high humidity (RH $\geq 80 \%{ }^{16}$. Exposure to high humidity was found to cause changes in both the diffraction efficiency and the Bragg selectivity curve of these holographic transmission gratings. Reversibility of the observed changes at high humidity strongly depended on temperature and was confirmed for temperatures below $15{ }^{\circ} \mathrm{C}$. The temperature limit of reversible/irreversible changes was established to correlate with the freezing/melting temperature range of one of the photopolymer components - triethanolamine. The ability of the transmission gratings to respond to high humidity is beneficial for the development of irreversible humidity holographic sensors, in particular for scenarios where increased temperature is also problematic e.g. food storage. 
The humidity induced changes in the properties of both transmission and reflection slanted gratings recorded in an acrylamide based photopolymer has been quantitatively analysed ${ }^{17}$ and the theoretical model of swelling dynamics has been proposed ${ }^{18}$.

In this study the capability to tune the temperature and relative humidity response of a holographic grating by compositional changes in the photopolymer layer has been explored. Two main components have been varied - the type of monomer (acrylamide versus diacetone acrylamide) and the type of initiator (triethanolamine versus $N$-phenylglycine). The two monomers were chosen because it was previously observed that gratings recorded in photopolymer layers containing acrylamide and diacetone acrylamide have very different holographic and mechanical properties ${ }^{19}$ which can influence on humidity and temperature sensitivity of the material. The two initiators were chosen because it was previously found that photopolymer layers containing triethanolamine are less dense and more permeable to water vapour and as a result the properties of photopolymer-based holographic gratings are humidity-dependant ${ }^{16,17}$. Whereas, photopolymer layers containing $\mathrm{N}$-phenylglycine are robust and the photopolymer-based holographic gratings are irresponsive to humidity changes up to $\mathrm{RH}=70 \%{ }^{20,21}$.

The first aim of this work was to study the humidity sensitivity of volume phase slanted gratings recorded in a non-toxic and environmentally compatible photopolymer containing diacetone acrylamide as the main monomer and triethanolamine as a photoinitiator. Comparative analysis of the humidity response of diacetone acrylamide-based gratings and acrylamidebased gratings has been carried out. Application of the diacetone acrylamide-based photopolymer as a humidity-responsive material for the development of holographic humidity sensors is also discussed.

As known, photopolymers in general have a low glass transition temperature and a relatively large coefficient of thermal expansion. Temperature variation induces grating detuning effects via changes in refractive index and the physical dimensions of the photopolymer layers ${ }^{22}$. Photopolymers that exhibit a mechanical response to temperature change are required for holographic temperature sensor development. Volume changes with temperature may be implemented as a sensing mechanism in holographic temperature sensors. However, previous explorations of the temperature response of acrylamide-based photopolymers are limited. The investigation of the reflection grating response to temperature in the range of $15-60{ }^{\circ} \mathrm{C}$ showed that the temperature response highly depends on the relative humidity level ${ }^{13}$.

The second aim of this work was to study the temperature sensitivity of volume phase slanted gratings recorded in photopolymer containing either acrylamide or diacetone acrylamide as monomer and either triethanolamine or $N$ phenylglycine as an initiator. The potential application of photopolymers as a thermo-responsive material for the development of holographic temperature sensors is discussed.

\section{BACKGROUND}

There are two types of holographic sensors depending on the recording geometry of the holographic grating utilized. The first type is a sensor based on a transmission holographic grating. This sensor can change its transmittance when interaction with a target analyte occurs. The sensor requires both a light source for its illumination and a photodetector to monitor a change in a signal level, such as a diffraction efficiency alteration or a variation of the diffracted light direction.

A volume phase transmission grating can be recorded using two laser beams when the photosensitive medium is placed in the region of overlap of two interfering wavefronts which are incident on the photosensitive medium from the same side. The interference pattern created by two light waves is recorded as a spatial modulation of the refractive index of the photosensitive medium. The volume phase grating regime corresponds to $Q \gg 1$, which is defined by the relation ${ }^{23}$

$$
Q=\frac{2 \pi \lambda d}{n \Lambda^{2}}
$$

where $\lambda$ is the wavelength of the recoding light, $d$ is the thickness of the grating, $n$ is the average refractive index of the medium and $\Lambda$ is the fringe spacing. The diffraction efficiency $(\eta)$ of a volume phase transmission grating at Bragg incidence is determined by the coupled wave theory ${ }^{24}$

$$
\eta=\sin ^{2}\left(\frac{\pi n_{1} d}{\lambda \cos \theta}\right),
$$

where $n_{l}$ is the refractive index modulation, $\lambda$ is the wavelength of the reconstructing beam, $\theta$ is the Bragg angle inside the photopolymer layer.

Exposure to an analyte leads to diffraction efficiency alterations $(\Delta \eta)$ caused by the effect of different parameters which can be determined by the differentiation of equation $(2)^{3}$ 


$$
\frac{\Delta \eta}{\eta}=\left|\frac{2}{\tan \left(\frac{\pi n_{1} d}{\lambda \cos \theta}\right)} \frac{\pi n_{1} d}{\lambda \cos \theta}\right|\left(\frac{\Delta n_{1}}{n_{1}}+\frac{\Delta d}{d}-\frac{\Delta \lambda}{\lambda}+\tan \theta \Delta \theta\right),
$$

where $\Delta n_{l}$ is the refractive index modulation change, $\Delta d$ is the thickness change, $\Delta \lambda$ is the variation of probe wavelength and $\Delta \theta$ is the Bragg angle shift.

The second type of holographic sensor is a sensor based on a reflection grating. The sensor operates via changes in the wavelength (colour) of the diffracted light under exposure to an analyte. These sensors are a focus of research as they can be used as visual indicators which can be easily interpreted by non-specialist. The reflection grating is recorded when two wavefronts reach the photosensitive medium from opposite sides. The direction of the maximum intensity of the light diffracted from the periodic photonic structure is determined by Bragg's law

$$
2 n \Lambda \sin \theta^{\prime}=\lambda,
$$

where $\theta^{\prime}$ is the Bragg angle which is defined by the angle between the incident beam and the planes of varying refractive index recorded in the material. Differentiation of equation (4) allows evaluating the contribution of different parameters on the spectral peak position of the diffracted light ${ }^{3}$

$$
\frac{\Delta \lambda}{\lambda}=\frac{\Delta n}{n}+\frac{\Delta \Lambda}{\Lambda}+\cot \theta \Delta \theta .
$$

Bragg angles in reflection geometry of recording are typically close to $90^{\circ}$. Thus, the contribution of the last term in equation (5) is usually negligible.

\subsection{Layer preparation}

\section{EXPERIMENTAL}

Three types of photopolymer layers containing different main monomers and photoinitiating systems have been used as a holographic recording material. The first type (photopolymer A) is an acrylamide-based photopolymer containing triethanolamine as a photoinitiator. Optimized compositions for the recording in transmission ${ }^{25}$ (photopolymer A_T) and reflection ${ }^{13}$ (photopolymer A_R) modes are presented in table 1. Lower concentration of triethanolamine which plays the roles of both a photoinitiator and a plasticizer is used in the composition for the recording in reflection mode. Less amount of triethanolamine allows decreasing the permeability of the material. It helps to reduce the diffusion of short polymer chains from bright to dark regions and the growth of polymer chains outside of bright fringes. This improves the spatial resolution of the material which is crucial for recording in reflection mode as the distance between fringes is much smaller than in case of transmission mode.

The second type (photopolymer B) is a version of the acrylamide-based photopolymer that uses $N$-phenylglycine as a photoinitiator ${ }^{20}$. Replacing triethanolamine with $N$-phenylglycine enables the development of robust photopolymer layers

\begin{tabular}{|c|c|c|c|c|}
\hline Chemical reagent & $\begin{array}{l}\text { Photopolymer A_T } \\
\text { (transmission) }\end{array}$ & $\begin{array}{l}\text { Photopolymer A_R } \\
\text { (reflection) }\end{array}$ & $\begin{array}{c}\text { Photopolymer B } \\
\text { (transmission/reflection) }\end{array}$ & $\begin{array}{l}\text { Photopolymer C } \\
\text { (transmission/reflection) }\end{array}$ \\
\hline Polyvinyl alcohol & $7.45 \% \mathrm{w} / \mathrm{v}$ & $7.95 \% \mathrm{w} / \mathrm{v}$ & $8.89 \% \mathrm{w} / \mathrm{v}$ & $7.4 \% \mathrm{w} / \mathrm{v}$ \\
\hline Acrylamide & $0.36 \mathrm{M}$ & $0.51 \mathrm{M}$ & $0.37 \mathrm{M}$ & --- \\
\hline $\begin{array}{l}\text { Diacetone } \\
\text { acrylamide }\end{array}$ & --- & --- & --- & $0.22 \mathrm{M}$ \\
\hline $\begin{array}{l}N, N^{\prime} \text {-Methylene } \\
\text { bisacrylamide }\end{array}$ & $0.055 \mathrm{M}$ & $0.074 \mathrm{M}$ & $0.034 \mathrm{M}$ & $0.048 \mathrm{M}$ \\
\hline Erythrosin B & $2.13 \times 10^{-4} \mathrm{M}$ & $1.7 \times 10^{-4} \mathrm{M}$ & $1.39 \times 10^{-4} \mathrm{M}$ & $1.85 \times 10^{-4} \mathrm{M}$ \\
\hline Triethanolamine & $0.64 \mathrm{M}$ & $0.51 \mathrm{M}$ & --- & $0.56 \mathrm{M}$ \\
\hline$N$-phenylglycine & --- & --- & $9.81 \times 10^{-3} \mathrm{M}$ & --- \\
\hline Glycerol & --- & --- & --- & $0.51 \mathrm{M}$ \\
\hline
\end{tabular}
which are non-sensitive to humidity.

Table 1. Photopolymer compositions 
The third type (photopolymer C) is a diacetone acrylamide-based photopolymer containing triethanolamine as a photoinitiator, and has previously been optimised for transmission mode of recording ${ }^{26,27}$. In addition to the components used in photopolymer A and photopolymer B, photopolymer C contains glycerol as a plasticizer. The addition of glycerol promotes diffusion of the relatively large diacetone acrylamide monomer molecules during holographic recording, resulting in higher diffraction efficiency than when glycerol is excluded. The addition of glycerol also improves the stability of the solid photopolymer layer ${ }^{27}$. The attempts to prepare samples containing diacetone acrylamide as the main monomer and $\mathrm{N}$-phenylglycine as a photoinitiator were not successful so far. The layers were not stable and were with poor optical quality. For that reason it was not possible to include results from the characterisation of gratings recorded in this composition. The same formulations of photopolymer B and photopolymer $\mathrm{C}$ were used for recording in both transmission and reflection mode (table 1). The further optimisation of these two photopolymer compositions for recording in reflection mode is currently under progress and will be reported elsewhere.

Photopolymer layers with the thickness of $60 \pm 5 \mu \mathrm{m}$ were prepared by deposition of photosensitive solutions on the levelled glass slides $\left(26 \times 76 \mathrm{~mm}^{2}\right)$ and dried for 24 hours in a dark room at $\mathrm{T}=21 \pm 2{ }^{\circ} \mathrm{C}$ and $\mathrm{RH}=30 \pm 5 \%$. The thickness of the dry layers was measured with a white light interferometric surface profiler MicroXAM S/N 8038.

\subsection{Holographic recording set-up}

Volume phase transmission and reflection gratings were recorded using a Nd:YVO 4 laser (532 nm). The holographic optical set-up used for recording in transmission mode is presented in figure 1a. A two-beam set-up with the incident angles of the recording beams of $4^{0}$ and $36^{\circ}$ (outside the layer), respectively to the normal to the layer surface, was utilized to record slanted transmission gratings with a slant angle of $13^{\circ}$ and a spatial frequency of 960 lines $/ \mathrm{mm}$. The total recording intensity was $5.5 \mathrm{~mW} / \mathrm{cm}^{2}$ and the recording time was $8 \mathrm{sec}$ for photopolymers A and C and $22 \mathrm{sec}$ for photopolymer B. These conditions were chosen in order to achieve similar starting diffraction efficiency in all three types of layers.

a)

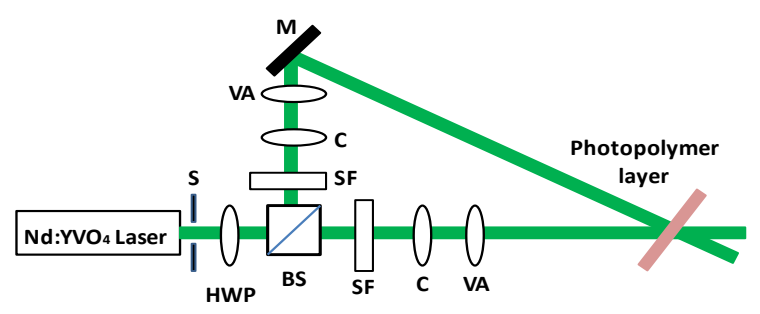

b)

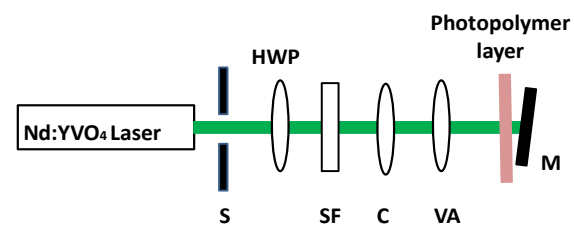

Figure 1. Experimental set-up for the recording of slanted transmission grating (a) and reflection grating (b): $\mathrm{S}$-electronic shutter; HWP - half-wave plate; BS - beam splitter; SF - spatial filter; C - collimator; VA-variable aperture; M - mirror.

The initial diffraction efficiency of the gratings was in the range of $37 \pm 3 \%$. In order to measure the diffraction efficiency of transmission gratings, the diffracted beam intensity was monitored at the Bragg angle using a $633 \mathrm{~nm}$ He-Ne laser. The diffraction efficiency of gratings was calculated as the ratio of the intensity of the diffracted beam and the intensity of the incident beam. After the recording, the gratings were exposed to UV-light for $18 \mathrm{~min}$ in order to polymerize all residual monomers using a UV Exposure unit (Mega Electronics, model 5503-11).

Reflection gratings with a slant angle of $5^{\circ}$ and a spatial frequency of approximately 5600 lines/mm were recorded with an exposure $120 \mathrm{~mJ} / \mathrm{cm}^{2}$ using the set-up presented in figure $1 \mathrm{~b}$.

\subsection{Humidity response testing}

The humidity response of gratings was investigated by measuring the diffraction efficiency of slanted transmission gratings and the spectral response of reflection gratings at different levels of relative humidity and at temperature of $21 \pm 1{ }^{\circ} \mathrm{C}$ (figure 2). Different humidity levels were obtained using a controlled environment chamber with humidity and temperature control system (Electro-tech system, model 5503-11). The chamber is equipped with ETS Series 5000 Microprocessor Controller which is utilized to precisely control the temperature and humidity, to provide accurate measurement and to monitor the chamber environment. The humidity level in the chamber can be reduced down to $10 \% \mathrm{RH}$ by the desiccant/pump dehumidification system. An ultrasonic humidification system allows the relative humidity to be increased up to $100 \%$. The heating system can increase the temperature from ambient temperature to $50{ }^{\circ} \mathrm{C}$. The chamber is able to maintain the relative humidity and temperature with accuracy of $\pm 2 \%$ and $\pm 1{ }^{\circ} \mathrm{C}$, respectively. 
a)

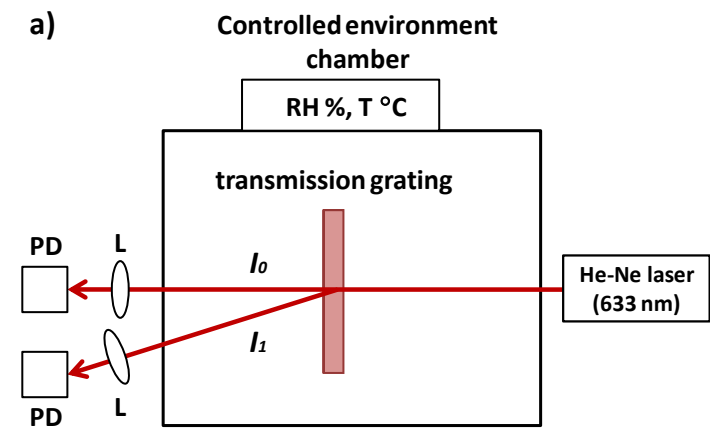

b)

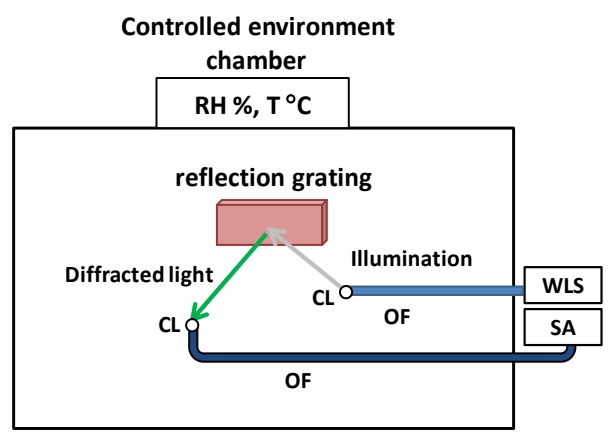

Figure 2. Schematic representation of the set-up for testing the humidity response of transmission grating (a) and reflection grating (b). L - lens; PD - photodetector; CL - collimating lens; OF - optical fibre; WLS - white light source; SA - spectral analyser.

During the test of the response to high humidity $(\mathrm{RH}=80-90 \%)$ it was observed that water vapour was condensing on the surface of the photopolymer layer causing intensity losses due to scattering. To minimize the inaccuracy caused by beam scattering due to water condensation on the photopolymer layer surface during the exposure to high humidity ( $>80$ $\%)$, intensities of transmitted $\left(I_{0}\right)$ and first-order diffracted $\left(I_{l}\right)$ beams were monitored simultaneously during the transmission grating test (figure 2a). The readings were taken after $30 \mathrm{~min}$ of humidity exposure to allow the samples to equilibrate with the surrounding conditions and the diffraction efficiency in this particular experiment was defined as $I_{1} /\left(I_{1}+I_{0}\right)$.

The optical set-up for the reflection grating test was assembled within the chamber to allow a spectrum diffracted by the sample to be characterised over a range of humidity levels (figure 2b). The probe light from a broad band light source (AvaLight HAL-S) was guided into the chamber by the fibre optic cable (Avantes FC-UV400-2). A collimating lens was attached to the fibre, which allowed for flexibility in adjustment of the incident angle. The diffracted light from the grating was coupled through a second fibre optic cable with the help of a collimating lens placed in front of the fibre to a spectral analyzer (Avantes AvaSpec-2048).

\subsection{Temperature response testing}

The temperature response of slanted transmission gratings was investigated by recording Bragg selectivity curves at different temperatures by a computer controlled system presented in figure 3 . Different temperatures were maintained by a temperature controlled plate (model MS100 with a $6 \mathrm{~mm}$ aperture, Linkam Instruments). The temperature controlled plate was able to hold a specified temperature to $\pm 0.1{ }^{\circ} \mathrm{C}$ from room temperature to $60{ }^{\circ} \mathrm{C}$. A $633 \mathrm{~nm}$ beam from a He-Ne laser was employed as a probe beam during the Bragg curve measurements. In order to record the Bragg curve, the grating was fixed on the temperature controlled plate and was placed on a rotation stage which was computer controlled via a motion controller (model Newport ESP300 with angular resolution of $0.001^{\circ}$ ). The intensity of the first-order diffracted beam was monitored by an optical power meter (Newport Model 840). LabVIEW software was used to plot the data of diffraction efficiency in real time as the incident angle of the probe beam was varied $\pm 3^{0}$ from the Bragg angle. The diffraction efficiency was defined as the ratio of the diffracted beam intensity and the incident beam intensity. The diffraction efficiency of the grating was estimated from the maximum of the Bragg selectivity curve. The readings were taken $5 \mathrm{~min}$ after the pre-set temperature was reached. This period of time was defined as the time required for reaching equilibrium of the sample temperature with the plate temperature. After 5 min no further change in the measured response was observed. 


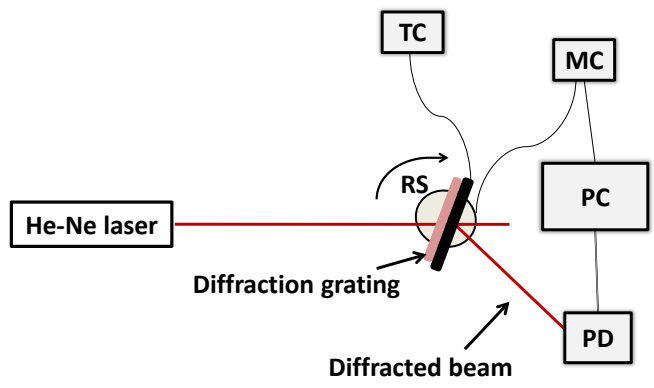

Figure 3. Set-up for the Bragg selectivity curve recording: RS - rotation stage; TC - temperature controller; MC - motion controller; $\mathrm{PC}$ - computer; PD - photodetector.

\subsection{Differential scanning calorimetry}

In order to analyze the amount of moisture desorbed from the photopolymer layers under heating, differential scanning calorimetry (DSC) was utilised. DSC measurements were performed using DSC-60 (Shimadzu). The measurements were conducted in aluminium pans by heating the samples at a rate of $2{ }^{\circ} \mathrm{C} / \mathrm{min}$ from 17 to $100{ }^{\circ} \mathrm{C}$. Prior to the DSC measurements, the samples were exposed to $\mathrm{RH}=70 \%$ at $\mathrm{T}=21 \pm 1{ }^{\circ} \mathrm{C}$ for an hour in the controlled environment chamber in order to saturate the photopolymer layers with the moisture.

\section{RESULTS AND DISCUSSION}

\subsection{Diffraction efficiency of slanted transmission gratings recorded in photopolymer $\mathrm{C}$ versus relative humidity}

The humidity response of slanted transmission gratings recorded in a diacetone acrylamide-based photopolymer (photopolymer C) was investigated by measuring the diffraction efficiency of the gratings in the relative humidity range of $20-90 \%$. During the experiment the temperature was kept constant at $21 \pm 1^{\circ} \mathrm{C}$. Figure 4 shows the relative change in the diffraction efficiency of gratings versus relative humidity. The experimental data for photopolymer $\mathrm{C}$ is compared with the experimental data for the acrylamide-based photopolymers (photopolymer A and photopolymer B).

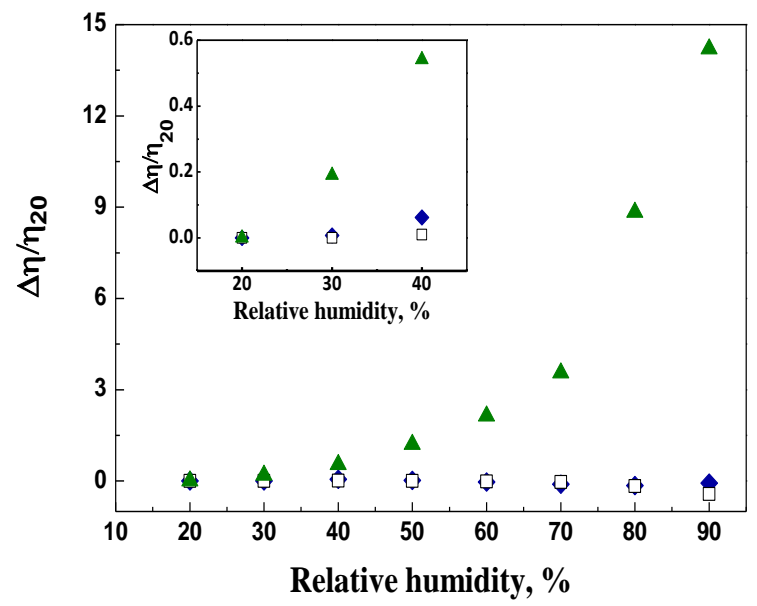

Figure 4. Relative changes in the on-Bragg diffraction efficiency of slanted transmission gratings recorded in photopolymer A ( $\downarrow)$, photopolymer B ( $\square)$ and photopolymer C $(\Delta)$ versus relative humidity. 
The relative change in the diffraction efficiency was calculated as follows. The difference of the diffraction efficiency measured at certain relative humidity and the diffraction efficiency measured at $\mathrm{RH}=20 \%(\Delta \eta)$ was normalized to the diffraction efficiency at $\mathrm{RH}=20 \%\left(\eta_{20}\right)$. For this particular experiment a slanted transmission grating with an initial diffraction efficiency of $6 \%$ was recorded in photopolymer C. As can be seen from figure 4 , changes in diffraction efficiency of photopolymer $\mathrm{C}$ caused by exposure to humidity were very significant (up to 14 times), i.e. increase of the diffraction efficiency from 6 to $84 \%$ was observed when the relative humidity changes from 20 to $90 \%$. The diffraction efficiency of slanted transmission gratings recorded in photopolymer A and B was relatively unchanged up to $70 \% \mathrm{RH}$ and it decreased for few percent above $70 \% \mathrm{RH}$.

According to equation (3), the significant increase of the diffraction efficiency of the transmission grating recorded in photopolymer $\mathrm{C}$ under humidity exposure can be caused by changes in several parameters. In order to fully understand the mechanism behind the observed change in diffraction efficiency, the effect of variation of each parameter must be considered. To estimate the effect of different parameters, the following experiment was carried out. Transmission gratings were exposed to relative humidity of $30 \%$ and $60 \%$. The diffraction efficiency, the Bragg angle and the thickness of the grating were monitored at $30 \%$ and $60 \% \mathrm{RH}$. The estimated contribution of different parameters is presented in table 2 . The first parameter is the Bragg angle shift. Our experimental results show that change in relative humidity from 30 to 60 $\%$ causes Bragg angle shift of $2^{0}$. The term describing the contribution of the Bragg angle shift is of order 0.006. The second parameter is the variation of probe wavelength. Since the probe wavelength used in this experiment was not varied the contribution of this term is zero. The third parameter is the thickness variation caused by humidity exposure. A thickness variation of 10 to $15 \%$ was found and this resulted in a thickness change term of order 0.1 . The fourth parameter is the change of the refractive index modulation. The refractive index modulation was calculated at $30 \%$ and $60 \% \mathrm{RH}$ using equation (2). The refractive index modulation change was found to be approximately $2 \times 10^{-4}$ and the term describing the contribution of the refractive index modulation alteration was of order 0.1 . Thus, it can be deduced that the main contributors to the diffraction efficiency alteration of transmission gratings recorded in photopolymer $\mathrm{C}$ are thickness and refractive index modulation changes. This means that the diffraction efficiency increase observed in gratings recorded in photopolymer $\mathrm{C}$ due to humidity exposure is caused by increases in both the grating thickness and the refractive index modulation. An increase of the grating thickness under humidity exposure can be explained by the photopolymer layer swelling due to water absorption. The refractive index modulation increase under humidity exposure may be due to the different ability of dark (i.e. unpolymerised) and bright (polymerised) regions to absorb water molecules due to its different porosity. One possible explanation is that the dark regions have higher porosity as monomers have lower density and as a result water molecules are mainly absorbed in these regions. As the average refractive index of the photopolymer is 1.5 , water $(n=1.33)$ absorption leads to a decrease of the refractive index in dark regions resulting in an overall increase of the refractive index modulation and, hence, the diffraction efficiency increases.

Table 2. Effect of different parameters on the diffraction efficiency change.

\begin{tabular}{|lllll|}
\hline Factor & Formula & $\begin{array}{l}\text { Estimated } \\
\text { contribution }\end{array}$ & $\begin{array}{l}\text { Physical process } \\
\text { responsible to this } \\
\text { change }\end{array}$ & $\begin{array}{l}\text { Significance in the } \\
\text { current experimental } \\
\text { conditions }\end{array}$ \\
\hline $\begin{array}{l}\text { Variation of the probe } \\
\text { beam incident angle }\end{array}$ & $\tan \theta \Delta \theta$ & 0.006 & $\begin{array}{l}\text { Spatial period increase } \\
\text { due to swelling }\end{array}$ & Minor \\
$\begin{array}{l}\text { Variation of probe } \\
\text { wavelength }\end{array}$ & $\Delta \lambda / \lambda$ & 0 & $\begin{array}{l}\text { Probe beam } \\
\text { wavelength was kept } \\
\text { constant in this study }\end{array}$ & NA \\
Thickness variation & $\Delta d / d$ & 0.1 & Swelling of the layer & High \\
$\begin{array}{l}\text { Change in the refractive } \\
\text { index modulation }\end{array}$ & $\Delta n_{1} / n_{1}$ & 0.1 & $\begin{array}{l}\text { Decrease of density in } \\
\text { dark regions }\end{array}$ & High \\
\hline
\end{tabular}

The experimental results reveal that photopolymer $\mathrm{C}$ has high sensitivity to humidity even at low relative humidity of 20 $-40 \%$ (figure 4). As seen from figure 4, a 54\% increase of the diffraction efficiency of photopolymer $\mathrm{C}$ was observed in this range, whereas the diffraction efficiency of photopolymer A and B was increased by few percent only. As discussed in section 3.1, photopolymer C contains the monomer diacetone acrylamide as well as small amounts of glycerol, both of which are known hygroscopic chemicals which readily absorb water ${ }^{28}$. Increased water absorption would account for the observed large increase in grating diffraction efficiency due to greater changes in grating thickness and refractive index modulation.

Thus, due to its high sensitivity to humidity, the diacetone acrylamide-based photopolymer can be utilized for the development of sensors for humidity monitoring in the relative humidity range from 20 to $90 \%$ and constant temperature. 
It should be noted that during humidity monitoring it is necessary to keep the temperature unchanged as photopolymer C is also sensitive to temperature variation (section 4.4). In contrast, photopolymers A and B are less sensitive to humidity, which is highly desirable for many holographic applications including holographic optical elements for solar applications and holographic security labels. The reversibility of the observed changes in photopolymer $\mathrm{C}$ was confirmed at RH $\leq 60$ $\%$ and will be further discussed in section 4.2 .

\subsection{Reversibility of the humidity induced changes in properties of photopolymer $\mathrm{C}$}

The reversibility of the effect of elevated humidity on gratings recorded in photopolymer $\mathrm{C}$ has been characterised. Investigation of the reversibility of humidity induced changes in photopolymer A and photopolymer B was carried out previously ${ }^{10,16,21}$. The reversibility for photopolymer A and photopolymer B was confirmed after exposure to $\mathrm{RH} \leq 70 \%$ and $\mathrm{RH} \leq 90 \%$, respectively, for $60 \mathrm{~min}$ at $\mathrm{T}=21 \pm 1{ }^{\circ} \mathrm{C}$. Exposure to high humidity $(\mathrm{RH}=80-90 \%)$ of photopolymer A for $60 \mathrm{~min}$ at $\mathrm{T}=21 \pm 1{ }^{\circ} \mathrm{C}$ caused irreversible decrease of the diffraction efficiency and dimensional changes in the layer.

In order to investigate the reversibility of the observed increase of the diffraction efficiency of photopolymer $\mathrm{C}$ during exposure to humidity, transmission gratings were exposed to $\mathrm{RH}=60 \%, 70 \%, 80 \%$ and $90 \%$ at $\mathrm{T}=21 \pm 1{ }^{\circ} \mathrm{C}$ for 60 min and left to recover at $\mathrm{T}=21 \pm 1{ }^{\circ} \mathrm{C}$ and $\mathrm{RH}=40-45 \%$ for 24 hours. Each grating was characterized by measuring its diffraction efficiency before and after exposure to humidity. The relative humidity during these measurements was in the range of $40-45 \%$.

Bragg selectivity curves of the grating before, during and 24 hours after exposure to $\mathrm{RH}=60 \%$ are presented on figure 5 . The diffraction efficiency of gratings under exposure to $60 \% \mathrm{RH}$ increases from 33 to $56 \%$ and the observed increase is in good agreement with the experimental data presented in figure 4 . After relaxation at $40 \% \mathrm{RH}$ the diffraction efficiency and the Bragg angle were found to be reversible. Peak of the diffraction efficiency was reversible to within $-0.1^{0}$ of its original position. The thickness of the photopolymer layer before and 24 hours after exposure to RH $=60 \%$ was the same. Thus, the increase of the diffraction efficiency observed during the exposure to humidity of $60 \% \mathrm{RH}$ is reversible.

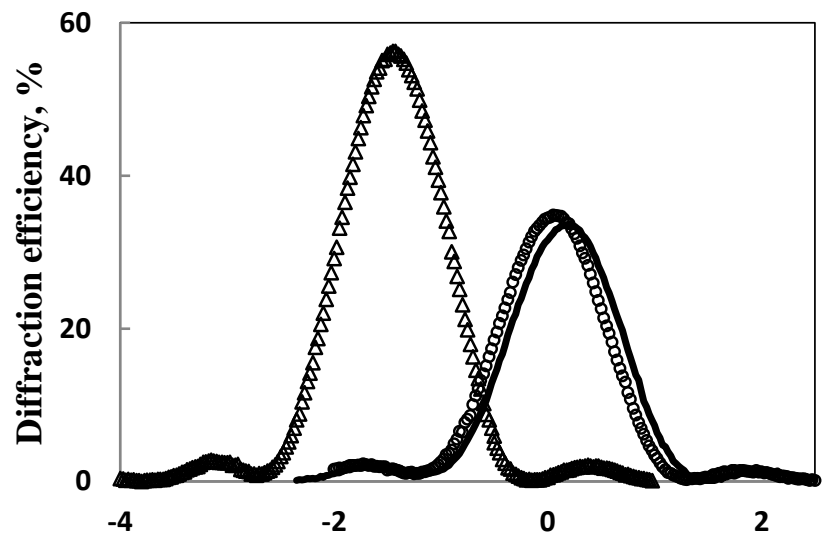

Deviation from Bragg angle, degree

Figure 5. Bragg selectivity curve for photopolymer $\mathrm{C}$ before (solid line), during ( $\Delta$ ) and 24 hours after (o) exposure to $\mathrm{RH}=60 \%$ at $\mathrm{T}$ $=21 \pm 1{ }^{0} \mathrm{C}$ for $60 \mathrm{~min}$.

Experimental data for the gratings exposed to $70 \%, 80 \%$ and $90 \% \mathrm{RH}$ for different amount of time are presented in figure 6. To describe humidity induced changes, normalized diffraction efficiency was calculated as the ratio of the diffraction efficiency measured after exposure to humidity and the diffraction efficiency measured before exposure to humidity. 


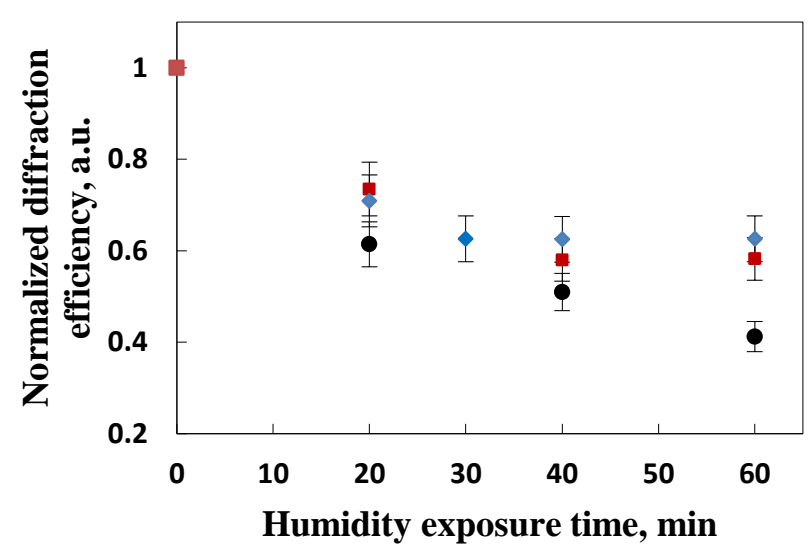

Figure 6. Normalized diffraction efficiency for photopolymer C after exposure to the relative humidity of $70 \%$ ( $\downarrow), 80 \%$ (घ) and 90 $\%(\bullet)$ versus humidity exposure time.

As can be seen from figure 6, exposure to humidity of $70 \%, 80 \%, 90 \%$ RH for 20 min causes irreversible decrease of the diffraction efficiency.

No dependence of the normalised diffraction efficiency on the time of exposure to 70 and $80 \%$ RH was observed above 30 min. Maximum decrease of the diffraction efficiency and its strongest dependence on time of exposure to high humidity was detected for $90 \% \mathrm{RH}$. One possible explanation for the observed irreversible decrease of the diffraction efficiency can be an irreversible decrease in the thickness of the grating. During humidity exposure, expansion of the photopolymer layer in the direction parallel to the grating vector was noticed. In order to evaluate the thickness change caused by humidity exposure, normalized thickness was calculated as the ratio of the thickness measured after and before humidity exposure. The relative humidity during thickness measurements was in the range of $40-45 \%$. Figure 7 shows that exposure to humidity of 70, 80 and $90 \% \mathrm{RH}$ leads to irreversible decrease of grating thickness.

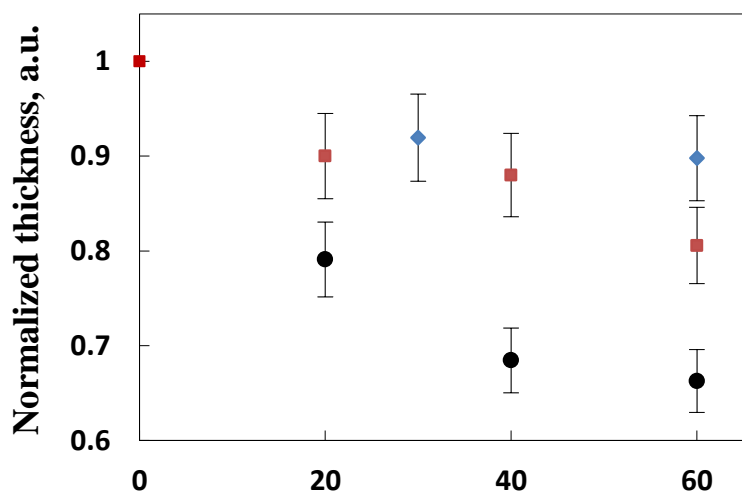

Humidity exposure time, min

Figure 7. Normalized thickness after exposure to the relative humidity of $70 \%(\bullet), 80 \%(\bullet)$ and $90 \%(\bullet)$ versus humidity exposure time.

Our estimations of the thickness utilising formula (2) reveal that the observed irreversible decrease of the diffraction efficiency can't be explained by irreversible decrease of the thickness only. This implies that at high humidity, irreversible decrease of the refractive index modulation also occurs. One possible explanation is follow. When the humidity is very high, the layers become close to a liquid state. The absorption of water allows for reduction in the degree of separation of the spatially varying regions of monomer molecules and polymer chains, i.e. the polymer chains and molecules become less rigidly fixed and move around, which would reduce the refractive index modulation.

Thus, photopolymer $\mathrm{C}$ can be utilized for the development of reversible sensors for continuous humidity monitoring in the range from 20 to $60 \% \mathrm{RH}$ at constant temperature. The photonic structure within photopolymer $\mathrm{C}$ changes its optical properties irreversibly in response to changes in the level of relative humidity above $60 \% \mathrm{RH}$, allowing for the creation of irreversible humidity holographic sensors with an operation range of $\mathrm{RH}>60 \%$ at constant temperature.

\subsection{Spectral response of reflection gratings at different levels of relative humidity}


Characterisation of the humidity response of reflection gratings recorded in photopolymer layers was carried out by monitoring the position of the peak intensity in the spectral response of reflection gratings in the range of $20-80 \% \mathrm{RH}$. During the experiment the temperature was kept constant at $21 \pm 1{ }^{\circ} \mathrm{C}$. The relative change in the wavelength of the diffracted light was calculated as the difference between the wavelength of the diffracted light measured at certain relative humidity and the wavelength of the diffracted light measured at $\mathrm{RH}=20 \%(\Delta \lambda)$, normalized to the wavelength of the diffracted light at $\mathrm{RH}=20 \%\left(\lambda_{20}\right)$. Relative changes in the wavelength of the diffracted light versus relative humidity are presented in figure 8 .

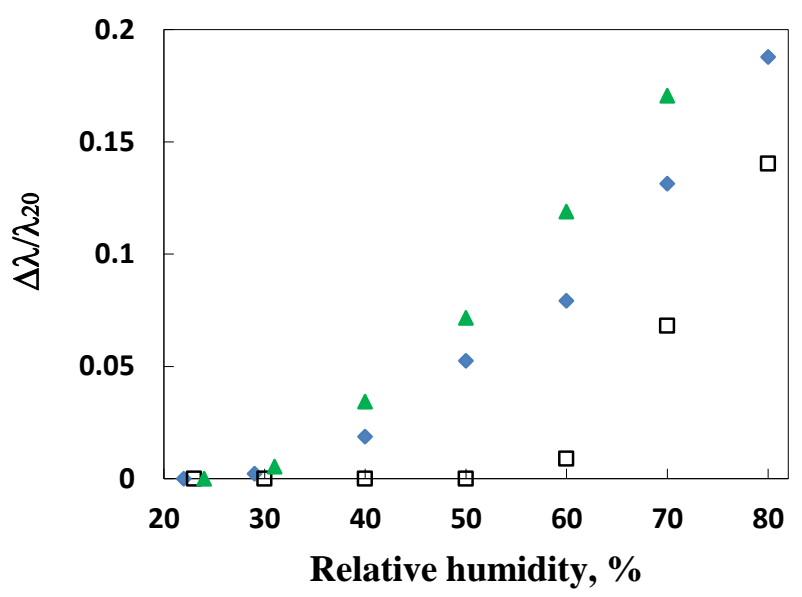

Figure 8. Spectral response of reflection gratings recorded in photopolymer A ( $\diamond)$, photopolymer B ( $\square)$ and photopolymer C ( $\Delta$ ) versus relative humidity.

Photopolymer A demonstrates a non-linear response over the studied humidity range, and the experimental data are in good agreement with the previously reported investigation ${ }^{13}$. Photopolymer B as expected is relatively unresponsive to variations in relative humidity below the $60 \% \mathrm{RH}$ threshold but has a close to liner response at $\mathrm{RH}>60 \%$. Photopolymer $\mathrm{C}$ displays significant sensitivity to relative humidity, particularly noticeable in the range of $40-70 \%$ RH. For a relative humidity change from 20 to $70 \%$, the wavelength shift of the diffracted light from the gratings recorded in photopolymer A, photopolymer B and photopolymer $\mathrm{C}$ were found to be $64 \pm 1 \mathrm{~nm}, 35 \pm 1 \mathrm{~nm}$ and $83 \pm 1 \mathrm{~nm}$, respectively. After one hour relaxation period at $20 \% \mathrm{RH}$ the shift in the position of the maximum intensity in the spectral response of each photopolymer layer was found to be reversible to within $4.8 \pm 2 \mathrm{~nm}$ of its spectral peaks original position.

According to formula (5), the main reasons for humidity response of the reflection grating are the refractive index variation and the spatial period alteration. When water vapour is absorbed, the photopolymer layer swells leading to an increase of the spatial period of the reflection grating recorded in the layer. It results in a decrease of the density of the photopolymer and, hence, its refractive index. Previously, it has been shown ${ }^{13}$ that changes in grating period is the main contributor to the wavelength shift.

The results demonstrate that photopolymers A, B and C can all be used for the development of holographic humidity sensors operating in reflection mode and visibly changing colour when reconstructed in white light. The operation range of the humidity sensor can be tuned by variation of the photopolymer composition.

\subsection{Temperature response of slanted transmission gratings}

The temperature response of slanted transmission gratings recorded in the different photopolymers was investigated by recording Bragg selectivity curves and monitoring the position of the Bragg angle in the temperature range of $20-60{ }^{\circ} \mathrm{C}$. Figure 9 presents the experimental data of the Bragg angle shift for photopolymer A, B and C. It should be noted that according to the recording geometry, the Bragg angle shift to a bigger value corresponds to the shrinkage of the layer in the direction perpendicular to the grating vector. 


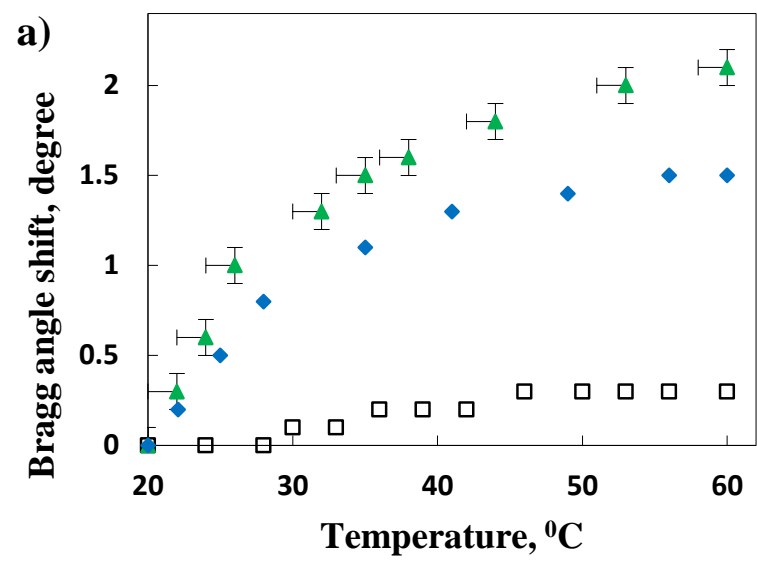

Figure 9. Temperature dependence of the Bragg angle shift of slanted transmission gratings recorded in photopolymer A ( $\diamond)$

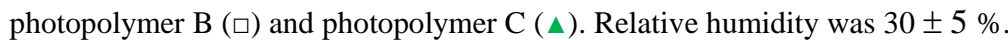

As shown in figure 9, in the case of photopolymer layers containing triethanolamine (photopolymer A and photopolymer C) exposure to temperature causes shrinkage of the layers leading to the Bragg angle shift up to $2^{0}$, whereas photopolymer B containing $N$-phenylglycine exhibits the lower response to temperature changes and the Bragg angle shift up to $0.3^{0}$ is observed. Analysis of the three types of layers carried out by DSC showed that the different behaviour of the Bragg angle shift for the different photopolymer layers can be explained by different ability of the three photopolymers to release water due to increase in temperature. DSC thermograms of photopolymers presented in figure 10 reveal that photopolymer A and photopolymer $\mathrm{C}$ have transitions with a distinct loss of weight due to drying in the temperature range of $20-55{ }^{\circ} \mathrm{C}$. Thus, drying of the layer causes its shrinkage, and, hence, the Bragg angle shift is observed (figure 9). In photopolymer B, the evaporation process is not so intensive leading to a smaller Bragg angle shift in this temperature range.

As outlined in section 4.1, photopolymer $\mathrm{C}$ is a highly humidity-sensitive material suitable for humidity sensor development. In order to eliminate effects due to humidity and to analyse only the thermal effect on the properties of the holographic gratings recorded in this photopolymer, the temperature response of sealed layers was investigated. Sealed samples were prepared by sealing the photopolymer layer on the glass substrate between two polyester films with a thickness of $0.05 \mathrm{~mm}$. Figure 11 shows that the behaviour of the Bragg angle shift of sealed and unsealed samples is fully opposite, i.e. exposure to temperature causes swelling of sealed layer and shrinkage of unsealed layer. Swelling of the sealed layer can be explained by relatively large thermal expansion of photopolymer $\mathrm{C}$, a property which is inherent to polymer materials with a low glass transition temperature.

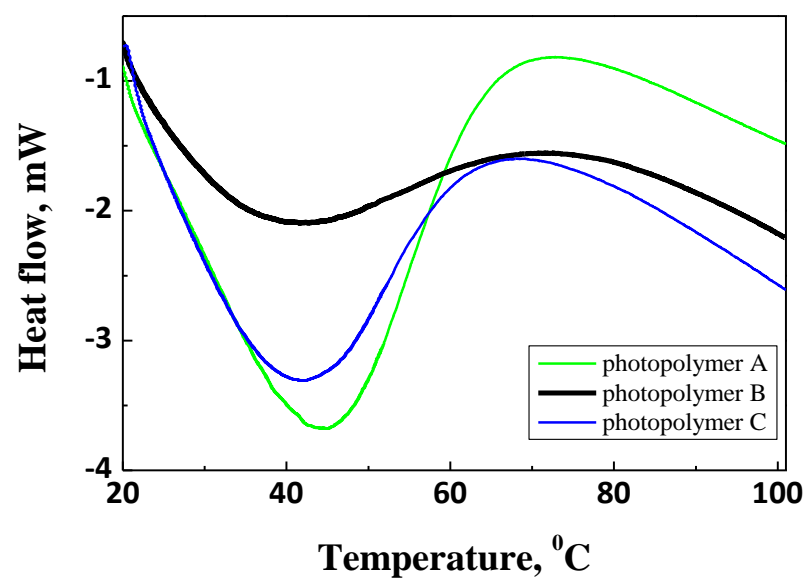

Figure 10. DSC thermograms of photopolymers. 


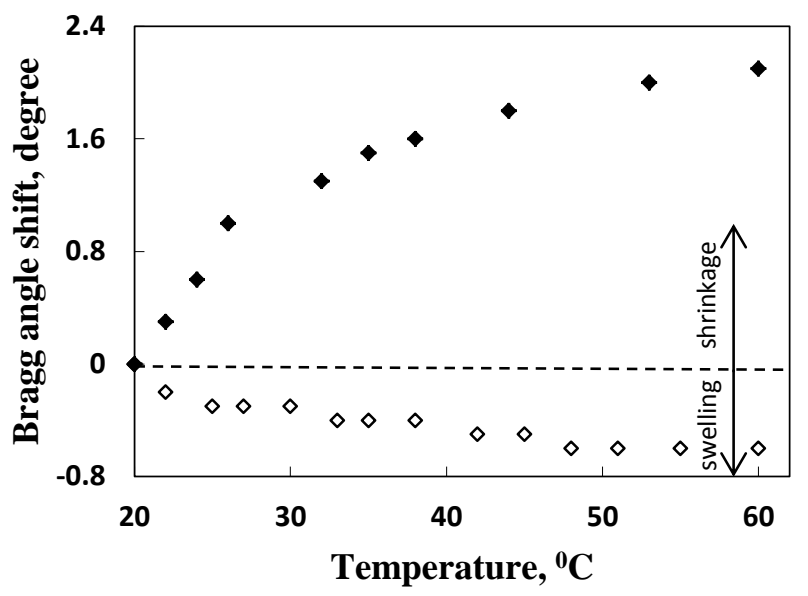

Figure 11. Temperature dependence of the Bragg angle shift in photopolymer C: unsealed sample $(\bullet)$ and sealed sample $(\diamond)$.

The temperature dependence of normalized diffraction efficiency of slanted transmission gratings recorded in unsealed and sealed layers for all three photopolymers is presented in figure $12 \mathrm{a}$ and $12 \mathrm{~b}$, respectively. Normalized diffraction efficiency was calculated as the ratio of the diffraction efficiency measured at certain temperature and the diffraction efficiency measured at the start of the experiment at $\mathrm{T}=20{ }^{\circ} \mathrm{C}$.
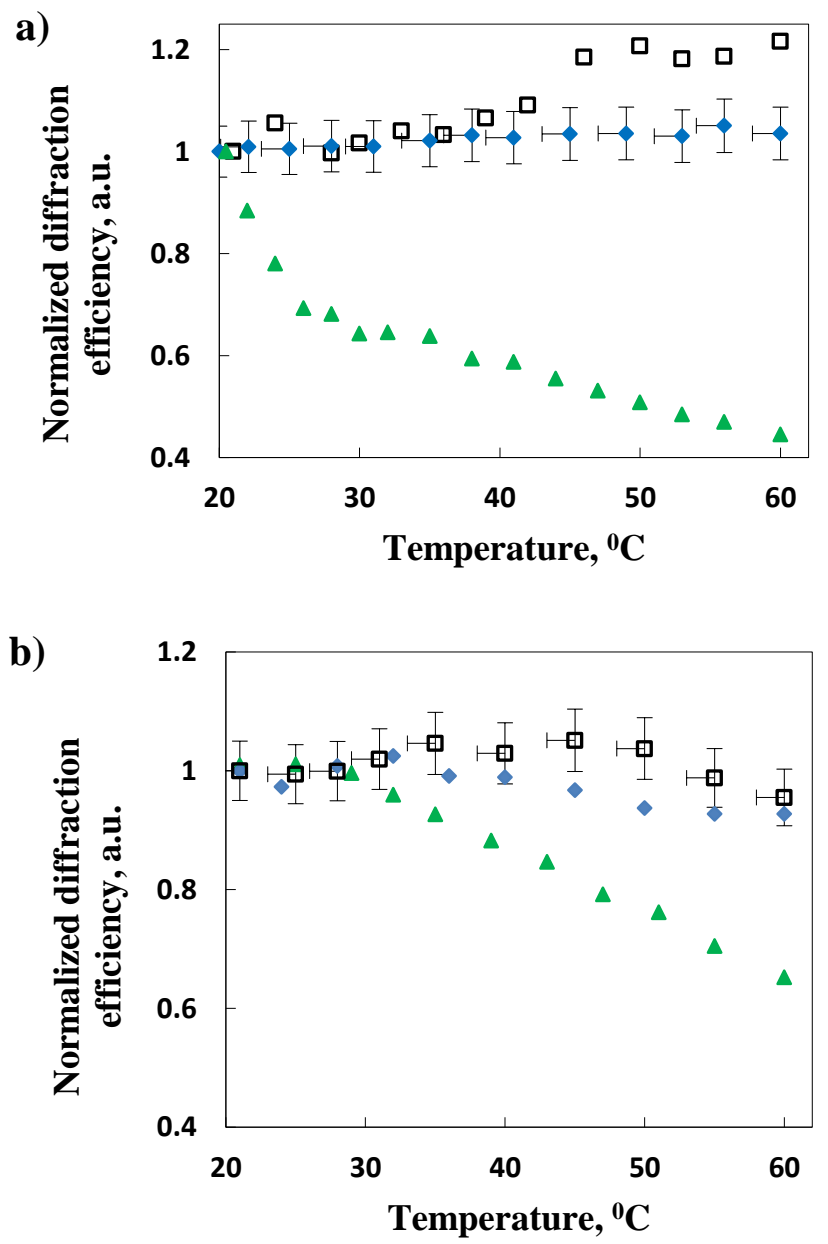

Figure 12. Temperature dependence of normalized diffraction efficiency of slanted transmission gratings recorded in unsealed (a) and sealed (b) photopolymer layers: $(\diamond)$ - photopolymer A, ( $)$ - photopolymer B and ( $\Delta)$ - photopolymer C. 
As seen from figure 12a, the diffraction efficiency of unsealed photopolymer A layers is unchanged in the temperature range from 20 to $60{ }^{\circ} \mathrm{C}$. Photopolymer $\mathrm{B}$ is unresponsive to temperature change below $40{ }^{\circ} \mathrm{C}$ and shows $20 \%$ increase in diffraction efficiency above $40{ }^{\circ} \mathrm{C}$. Significant decrease of the diffraction efficiency of photopolymer $\mathrm{C}$ is observed in the range of 20 to $60{ }^{\circ} \mathrm{C}$. Similarly to the humidity exposure tests, the observed temperature dependence of the normalized diffraction efficiency depends greatly on changes in the grating refractive index modulation. It is expected that there are two main processes which have an effect on the refractive index modulation alterations. The first contributor is desorption of adsorbed moisture due to elevated temperature. This predominantly takes place in the dark regions as these areas are less dense and therefore absorb more water. Water desorption leads to increase of the refractive index in the dark regions and, hence, decrease of the refractive index modulation. The second contributor is thermal expansion which leads to a decrease of the polymer density. Depending on which region (i.e. the dark or bright region) expands to a greater extent, the diffraction efficiency is increased/decreased with respect to temperature change. This theory matches with the results obtained in section 4.1, which showed that composition $\mathrm{C}$ is significantly more prone to water absorption than photopolymer $\mathrm{A}$ and $\mathrm{B}$. Therefore, a greater response to elevated temperature is expected for photopolymer $\mathrm{C}$ as it is more likely to experience stronger water desorption effects than either photopolymer A or B.

Application of the sealing technique allowed for observation of changes in the diffraction efficiency caused by thermal expansion (figure 12b). Photopolymer $\mathrm{C}$ reveals temperature sensitivity above $30{ }^{\circ} \mathrm{C}$ demonstrating its potential suitability for the development of holographic temperature sensors. Photopolymer A and photopolymer B is relatively unresponsive to temperature changes in the range of $20-60{ }^{\circ} \mathrm{C}$. A photopolymer material which is stable over a large temperature range is highly desirable for many holographic applications.

\section{CONCLUSION}

The humidity and temperature sensitivity of slanted holographic gratings recorded in photopolymers containing acrylamide and diacetone acrylamide as monomers, and triethanolamine and $\mathrm{N}$-phenylglycine as photoinitiators was investigated. It has been demonstrated that the response of the photopolymer-based holographic gratings to relative humidity and temperature can be tuned by variation of the photopolymer composition.

Characterization of the humidity response of photopolymer-based gratings was carried out in the range of $20-90 \%$ RH. A strong humidity dependence of the diffraction efficiency of diacetone acrylamide photopolymer-based transmission gratings was observed. Reversibility of humidity induced changes is confirmed at $\mathrm{RH} \leq 60 \%$. Thus, the response to humidity of the diacetone acrylamide-based gratings can be utilized for the development of reversible sensors for continuous humidity monitoring in the range of 20-60\% RH and constant temperature. Also, irreversible decrease of the diffraction efficiency caused by exposure to $\mathrm{RH}>60 \%$ is particularly useful for fabrication of irreversible humidity indicators suitable for incorporation in packaging for electronic goods, for example.

The humidity dependence of the spectral response of reflection gratings showed that photopolymers containing triethanolamine are more hydrophilic than photopolymers containing $N$-phenylglycine. Photopolymers containing triethanolamine demonstrated a non-linear response over the humidity range under study with a noticeable increase in sensitivity in the range of $40-70 \% \mathrm{RH}$. The photopolymer layers containing $N$-phenylglycine had very limited response to variations in relative humidity below $60 \% \mathrm{RH}$ and had a similar trend of response to photopolymers containing triethanolamine above $60 \% \mathrm{RH}$ but show less layer swelling. Thus, photopolymers can be used for the development of white light visible, reflection mode holographic humidity indicators and its operation range can be tuned by using photopolymers with different composition.

The temperature response of slanted transmission gratings was investigated in the temperature range of $20-60{ }^{\circ} \mathrm{C}$. The unsealed photopolymer layers containing $N$-phenylglycine were unresponsive to temperature changes below $30{ }^{\circ} \mathrm{C}$ and showed sensitivity to temperature above $30{ }^{\circ} \mathrm{C}$. In the temperature range from 30 to $60{ }^{\circ} \mathrm{C}$ the diffraction efficiency changed from 12 to $14 \%$ and the Bragg angle shift of $0.3^{\circ}$ was observed. Exposure of the diacetone acrylamide based-photopolymer layers containing triethanolamine to elevated temperature leads to the significant decrease of the diffraction efficiency up to 2 times and the Bragg angle shift up to $2^{0}$ due to the shrinkage of the unsealed layer caused by water evaporation. Application of a sealing technique allowed for observation of swelling of the diacetone acrylamide-based photopolymer layer due to its thermal expansion. The results demonstrate an effective approach to obtaining photopolymer-based gratings with tuneable temperature sensitivity and possibility to exploit photopolymers as a thermo-responsive material for the development of temperature holographic sensors.

The authors would like to acknowledge the financial support provided by the Irish Research Council Embark Initiative and Ulysses Programme, the COST 1205 Action, and also thank the FOCAS Research Institute for providing research facilities. 


\section{REFERENCES}

Blyth, J., Lowe, C. R., Mayes, A. G. \& Millington, R. B. $\quad$ (Google Patents, 1999).

Kabilan, S. et al. Glucose-sensitive holographic sensors. Journal of Molecular Recognition 17, $162-$ 166, doi:10.1002/jmr.663 (2004).

Yetisen, A. K., Naydenova, I., da Cruz Vasconcellos, F., Blyth, J. \& Lowe, C. R. Holographic Sensors: Three-Dimensional Analyte-Sensitive Nanostructures and Their Applications. Chemical Reviews 114, 10654-10696, doi:10.1021/cr500116a (2014).

Bhatta, D., Christie, G., Madrigal-González, B., Blyth, J. \& Lowe, C. R. Holographic sensors for the detection of bacterial spores. Biosensors and Bioelectronics 23, 520-527, doi:http://dx.doi.org/10.1016/i.bios.2007.06.006 (2007).

Fuchs, Y., Kunath, S., Soppera, O., Haupt, K. \& Mayes, A. G. Molecularly Imprinted Silver-Halide Reflection Holograms for Label-Free Opto-Chemical Sensing. Advanced Functional Materials 24, 688694, doi:10.1002/adfm.201301454 (2014).

Kraiskii, A., Postnikov, V, Sultanov, T, Khamudilin,A. Holographic sensors for diagnostics of solution components. Quantum Electronics 40, 4 (2010).

Leite, E., Naydenova, I., Mintova, S., Leclercq, L. \& Toal, V. Photopolymerizable nanocomposites for holographic recording and sensor application. Appl. Opt. 49, 3652-3660, doi:10.1364/ao.49.003652 (2010).

Martínez-Hurtado, J. L., Davidson, C. A. B., Blyth, J. \& Lowe, C. R. Holographic Detection of Hydrocarbon Gases and Other Volatile Organic Compounds. Langmuir 26, 15694-15699, doi:10.1021/la102693m (2010).

Mihaylova, E., Cody, D., Naydenova, I., Martin, S. \& Toal, V. Research on Holographic Sensors and Novel Photopolymers at the Centre for Industrial and Egnineering Optics. (2013).

Naydenova, I., Jallapuram, R., Toal, V. \& Martin, S. A visual indication of environmental humidity using a color changing hologram recorded in a self-developing photopolymer. Applied Physics Letters 92, 031109, doi:doi:http://dx.doi.org/10.1063/1.2837454 (2008).

Yetisen, A. K. et al. Light-Directed Writing of Chemically Tunable Narrow-Band Holographic Sensors. Advanced Optical Materials 2, 250-254, doi:10.1002/adom.201300375 (2014).

Naydenova, I., Sherif, H., Martin, S., Jallapuram, R. \& Toal, V. (Google Patents, 2012).

Naydenova, I., Jallapuram, R., Toal, V. \& Martin, S. Characterisation of the humidity and temperature responses of a reflection hologram recorded in acrylamide-based photopolymer. Sensors and Actuators B: Chemical 139, 35-38, doi:http://dx.doi.org/10.1016/j.snb.2008.08.020 (2009). Bently, R. Handbook of Temperature Measurement. (Springer, 1998).

5 Chen, Z. \& Lu, C. Humidity Sensors: A Review of Materials and Mechanisms. Sensor Letters 3, 274295, doi:10.1166/sl.2005.045 (2005).

6 Mikulchyk, T., Martin, S. \& Naydenova, I. Humidity and temperature effect on properties of transmission gratings recorded in PVA/AA-based photopolymer layers. Journal of Optics 15, 105301 (2013).

1 Yu, D. et al. Holographic humidity response of slanted gratings in moisture-absorbing acrylamide photopolymer. Appl. Opt. 54, 6804-6812, doi:10.1364/ao.54.006804 (2015).

8 Liu, H., Yu, D., Mao, D., Geng, Y. \& Wang, W. Modeling swelling and absorption dynamics for holographic sensing in analytes sensitive photopolymer. Optics Communications 368, 95-104, doi:http://dx.doi.org/10.1016/j.optcom.2016.02.005 (2016).

Cody, D. Low-toxicity diacetone acrylamide-based photopolymer for applications in holography, Dublin Institute of Technology, (2014).

Gong, Q., Wang, S., Huang, M. \& Gan, F. A humidity-resistant highly sensitive holographic photopolymerizable dry film. Materials Letters 59, 2969-2972, doi:http://dx.doi.org/10.1016/j.matlet.2005.05.008 (2005). 
21 Mikulchyk, T., Martin, S. \& Naydenova, I. Investigation of the sensitivity to humidity of an acrylamide-based photopolymer containing $\mathrm{N}$-phenylglycine as a photoinitiator. Optical Materials 37, 810-815, doi:http://dx.doi.org/10.1016/i.optmat.2014.09.012 (2014).

22 Dhar, L. et al. Temperature-induced changes in photopolymer volume holograms. Applied Physics Letters 73, 1337-1339, doi:doi:http://dx.doi.org/10.1063/1.122375 (1998).

23 Phariseau, P. On the diffraction of light by progressive supersonic waves. Proc. Indian Acad. Sci. 44, 165-170, doi:10.1007/bf03046040 (1956).

24 Kogelnik, H. Coupled Wave Theory for Thick Hologram Gratings. The Bell System Technical Journal 48, 2909-2947 (1969).

25 Martin, S., Feely, C. A. \& Toal, V. Holographic recording characteristics of an acrylamide-based photopolymer. Appl. Opt. 36, 5757-5768, doi:10.1364/ao.36.005757 (1997).

26 Cody, D., Naydenova, I. \& Mihaylova, E. New non-toxic holographic photopolymer material. Journal of Optics 14, 015601 (2012).

27 Cody, D., Naydenova, I. \& Mihaylova, E. Effect of glycerol on a diacetone acrylamide-based holographic photopolymer material. Appl. Opt. 52, 489-494, doi:10.1364/ao.52.000489 (2013).

28 Material Safety Data Sheet $<$ http://www.sigmaaldrich.com/>1 